Respioilion

\title{
'Idiopathic' Effusions Get a Proper Name
}

\author{
Derick Nelson Jenkins Katie V. Bean Manpreet Singh Malik \\ Department of Internal Medicine, VCU Health, Richmond, Va., USA
}

A 46-year-old African American male with 2 years of idiopathic, symptomatic, and recurrent pleural and pericardial effusions, asthma, and chronic pericarditis presented with a 1-month history of progressive exertional dyspnea and lower-extremity edema.

Prior workup included a thoracentesis and pleural biopsy that revealed an exudative effusion and chronic pleuritis, respectively, but no definitive diagnosis.

His heart rate was 102 , respiratory rate 18 , and oxygen saturation $97 \%$ on room air. Physical examination was significant for muffled heart sounds, absence of jugular vein distention, absence of pulsus paradoxus, absence of murmur, clear breath sounds, trace of lower-extremity edema, and absence of rash.

Laboratory tests including complete blood count and chemistries were unremarkable.
Chest CT showed a moderate right pleural effusion and moderate-to-large pericardial effusion (see fig. 1). Transthoracic echocardiography showed a moderate pericardial effusion with mild hemodynamic compromise (see fig. 2). Cardiac MRI revealed a moderate-tolarge circumferential, simple-appearing pericardial effusion with features of hemodynamic compromise (dilated inferior vena cava and biatrial/right ventricular partial collapse) and small left and moderate right-sided pleural effusions.

A pericardial window was performed and fluid analysis revealed 889,888 red blood cells $/ \mathrm{mm}^{3}$ and 6,235 total nucleated cells $/ \mathrm{mm}^{3}$ with lymphocytic predominance.

What is your diagnosis?

\section{KARGER}

E-Mail karger@karger.com www.karger.com/res
(C) 2016 S. Karger AG, Basel

0025-7931/16/0922-0114\$39.50/0
Derick Nelson Jenkins

Department of Internal Medicine, VCU Health Sanger Hall, Suite 1-030, 1101 East Marshall Street Richmond, VA 23298-0663 (USA)

E-Mail derick.jenkins@ vcuhealth.org 


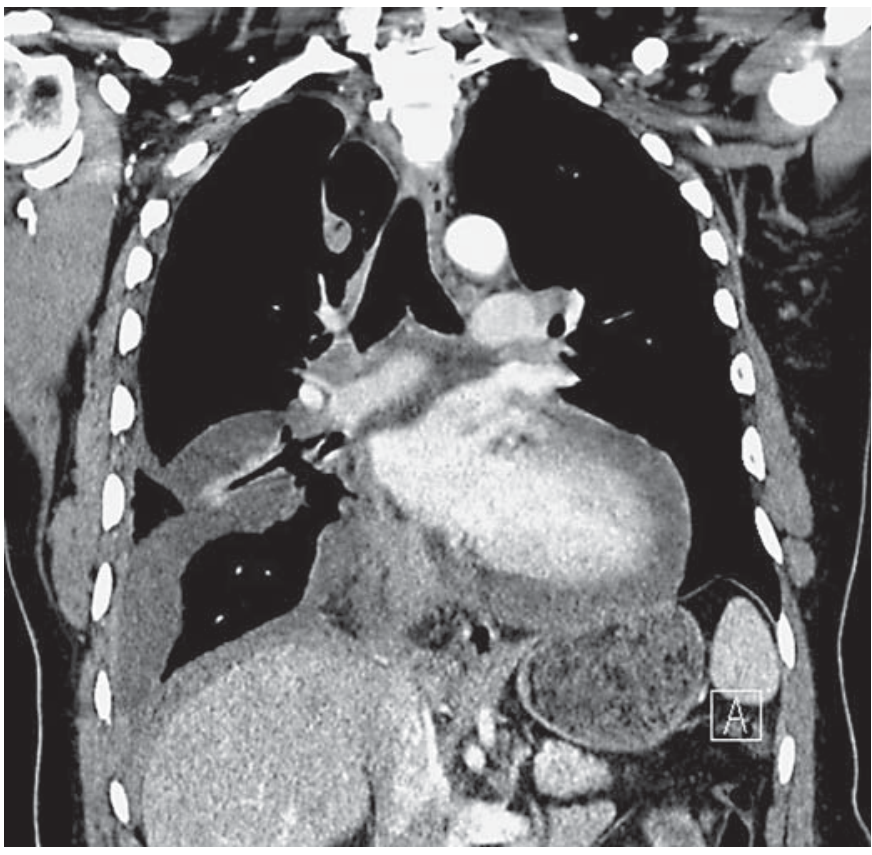

Fig. 1. Coronal CT demonstrating right pleural effusion and moderate-to-large pericardial effusion.

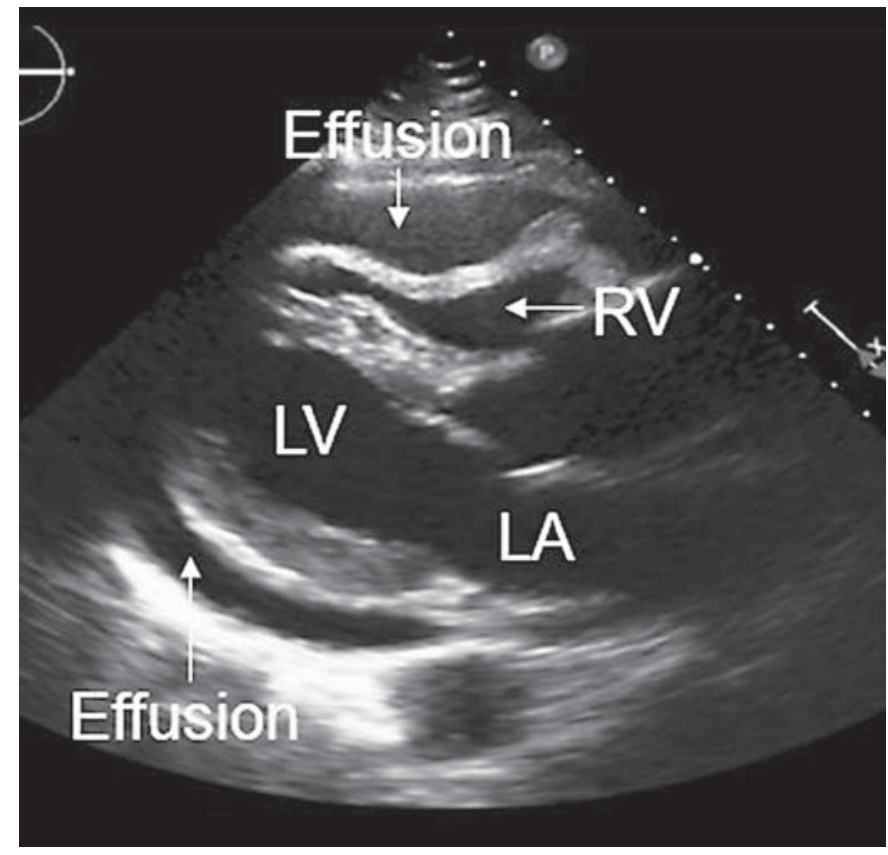

Fig. 2. Transthoracic echocardiogram demonstrating mild hemodynamic compromise during the relaxation phase. RV = Right ventricle; $\mathrm{LA}=$ left atrium; $\mathrm{LV}=$ left ventricle. 


\section{Diagnosis: Sarcoidosis}

Pericardial biopsy revealed chronic pericarditis and noncaseating granulomas (see fig. 3). Pericardial GMS (fungal) and AFB stains were negative as were fungal and AFB cultures. Interestingly, the patient had no history of ocular, dermatologic, gastrointestinal/hepatic, joint, neurologic, or myocardial involvement. He was started on prednisone with improvement of his symptoms and imaging.

Sarcoidosis is characterized by noncaseating granulomatous inflammation, and the exact cause of granuloma formation is unclear. This disease can have a highly variable presentation due to the multiple organ systems it affects and the multitude of responses the disease elicits. That being said, it commonly presents with nonspecific constitutional symptoms, dyspnea, cough, chest pain, skin involvement, and/or uveitis. It may even be asymptomatic with the diagnosis made following incidental radiographic findings [1], the most common of which is mediastinal lymphadenopathy. While it often affects the pulmonary and cardiac systems, presentation with both pericardial and pleural effusions is exceedingly rare. Review of the literature yielded only a few reported cases of sarcoidosis presenting with this combination of diagnoses [2-4].
Pleural effusions are common in medicine and can be found in a variety of disease processes including pulmonary, cardiac, hepatic, infectious, and oncologic maladies. Once the diagnosis of a pleural effusion is made, fluid testing allows for further subdivision into transudative or exudative processes. Transudative effusions are caused by a disruption in hydrostatic pressures and oncotic forces, whereas exudative effusions are due to inflammatory changes causing capillary leakage. The most common causes of exudative effusions are, in order of decreasing frequency, bacterial pneumonias, pulmonary embolism, and malignancy [5]. Sarcoidosis most commonly causes lymphocyte-predominant exudative effusions [6], as seen in our patient. Approximately 5\% of the patients with sarcoidosis develop pleural effusions [2], which may be present at the onset of the disease [2,7].

As pleural effusions, pericardial effusions have multiple causes. Some of the most commonly reported etiologies include malignancy, acute myocardial infarction, manipulation or disruption of the pericardium due to cardiac surgery or trauma, end-stage renal disease causing uremia, hypothyroidism, chest radiation, and autoimmune diseases [8]. One quarter of the patients with sarcoidosis have cardiac granulomas on autopsy, but cardiac

Fig. 3. Pericardial biopsy tissue microscopy showing chronic pericarditis and noncaseating granulomas.

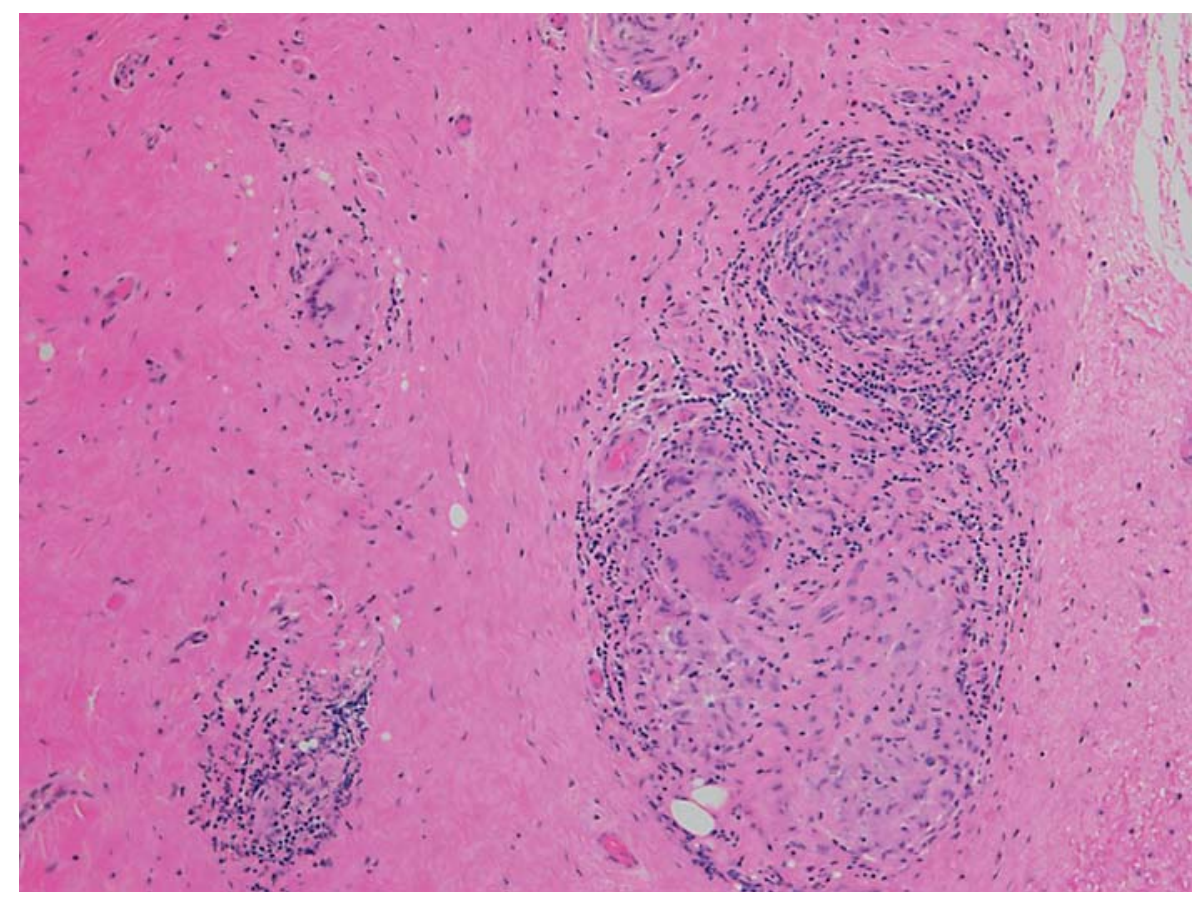


sarcoidosis only becomes clinically apparent in around $5 \%$ of the patients [9]. Sarcoidosis is reported in the literature as a cause of pericardial effusions; however, these are typically small and asymptomatic. One study of 81 patients with biopsy-proven sarcoidosis found that $21 \%$ of the patients who were asymptomatic from a cardiac standpoint had 'mild or moderate size pericardial accumulations' [10]. Sarcoidosis has rarely been reported to cause cardiac tamponade. A case report by Sangwan et al. [11] from 2010 commented that there were 11 case reports of this in the literature at that time. Notably, in the case of our patient, echocardiography showed some de- gree of hemodynamic compromise with partial rightventricle collapse during the relaxation phase of the cardiac cycle (see fig. 2).

Sarcoidosis can be difficult to diagnose as the presenting symptoms can be nonspecific and affect virtually any organ system. Further complicating this is the fact it can present in atypical ways, as with our patient who presented with recurrent pleural and pericardial effusions as well as chronic pericarditis. Sarcoidosis should be considered in the differential for patients with newly or previously diagnosed idiopathic pleural and pericardial effusions.

\section{References}

$1 \mathrm{Wu}$ JJ, Schiff KR: Sarcoidosis. Am Fam Physician 2004;70:312-322.

- Navaneethan SD, Venkatesh S, Shrivastava R, Mehta J, Israel R: Recurrent pleural and pericardial effusions due to sarcoidosis. PLoS Med 2005;2:e63.

3 Krawczyk I, Sedlaczek AM: A case of sarcoidosis with massive pleural and pericardial effusion (in Polish). Pneumonol Alergol Pol 1997; 65:81-85.

-4 Currie GP, Kerr K, Buchan K, Garg D: A rare cause of recurrent massive pericardial and pleural effusions. QJM 2008;101:989-990.
Saguil A, Wyrick K, Hallgren J: Diagnostic approach to pleural effusion. Am Fam Physician 2014;90:99-104.

6 Joshi S, Periwal P, Dogra V, Talwar D: Sarcoidosis as unusual cause of massive pleural effusion. Respir Med Case Rep 2015;16:143145.

7 Salazar A, Mana J, Corbella X, Vidaller A: Sarcoid pleural effusion: a report of two cases. Sarcoidosis 1994;11:135-137.
Sagristà-Sauleda J, Mercé AS, Soler-Soler J: Diagnosis and management of pericardial effusion. World J Cardiol 2011;3:135-143.

-9 Iannuzzi MC, Rybicki BA, Teirstein AS: Sarcoidosis. N Engl J Med 2007;357:2153-2165.

10 Angomachalelis N, Hourzamanis A, Salem N, et al: Pericardial effusion concomitant with specific heart muscle disease in systemic sarcoidosis. Postgrad Med J 1994;70:S8-S12.

11 Sangwan YS, Altaqi B, Ellithorpe DB, Mcllelan S, Parada NA: Sarcoidosis presenting as cardiac tamponade in a 30-year-old woman. Chest 2010;138:8A. 